\title{
Compensating for Pneumatic Distortion in Pressure Sensing Devices
}

Stephen A. Whitmore

Ames Research Center, Dryden Flight Research Facility, Edwards, California

Cornelius T. Leondes

Mechanical, Aerospace, and Nuclear Engineering Department

University of California, Los Angeles

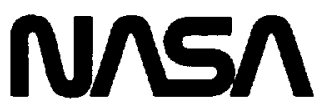

National Aeronautics and Space Administration Ames Research Center Dryden Flight Research Facility Edwards, California 93523-5000 


\title{
COMPENSATING FOR PNEUMATIC DISTORTION IN PRESSURE SENSING DEVICES
}

\author{
Stephen A. Whitmore* \\ NASA Ames Research Center \\ Dryden Flight Research Facility \\ Edwards, California \\ and \\ Cornelius T. Leondes** \\ Mechanical, Aerospace, and Nuclear Engineering Department \\ University of California, Los Angeles
}

\begin{abstract}
In this paper a technique of compensating for pneumatic distortion in pressure sensing devices is developed and verified. This compensation allows conventional pressure sensing technology to obtain improved unsteady pressure measurements. Pressure distortion caused by frictional attenuation and pneumatic resonance within the sensing system makes obtaining unsteady pressure measurements by conventional sensors difficult. Most distortion occurs within the pneumatic tubing which transmits pressure impulses from the aircraft's surface to the measurement transducer. To avoid pneumatic distortion, experiment designers mount the pressure sensor at the surface of the aircraft, (called in situ mounting). In situ transducers cannot always fit in the available space and sometimes pneumatic tubing must be run from the aircraft's surface to the pressure transducer. Research began at the National Aeronautics and Space Administration Ames Research Center Dryden Flight Research Facility, supported by the University of Califormia, Los Angeles, to develop a technique to measure unsteady pressure data using conventional pressure sensing technology. A pneumatic distortion model is reduced to a low-order, statevariable model retaining most of the dynamic characteristics of the full model. The reduced-order model is coupled with results from minimum variance estimation theory to develop an algorithm to compensate for the effects of pneumatic distortion. Both postflight and real-time algorithms are developed and evaluated using simulated and flight data.
\end{abstract}

\section{Nomenclature}

\section{Acronyms}

BVP boundary value problem

\footnotetext{
*Aerospace Engineer. Member AIAA.

* Professor. Member AIAA.

Copyright (C) 1989 by the American Institute of Acronautics and Astronautics, Inc. No copyright is asserted in the United States under Title 17. U.S. Code. The U.S. Govemment has a royalty-free license to exercise all rights under the copyright claimed herein for Govemmental purposes. All other rights are reserved by the copyright owner.
}
ESP
DC
electronically scanned pressure module direct current

\begin{tabular}{|c|c|}
\hline Symbols & \\
\hline$A_{\mathrm{c}}$ & cross-sectional area of pneumatic tubing \\
\hline$B$ & state equation input geometry matrix \\
\hline$C$ & observation matrix \\
\hline COV & covariance \\
\hline$c$ & sonic velocity \\
\hline$D$ & pressure tubing cross-sectional diameter \\
\hline$d x$ & spatial coordinate differential \\
\hline$G$ & stationary measurement error variance \\
\hline$G_{k+1}$ & measurement noise covariance matrix \\
\hline$G(1)$ & first diagonal component of $G_{k+1}$ \\
\hline$G(2)$ & second diagonal component of $G_{k+1}$ \\
\hline$g_{k+1}$ & measurement noise function \\
\hline$I$ & identity matrix \\
\hline$I$ & input to deconvolution algorithm \\
\hline$\hat{I}$ & deconvolution input estimate \\
\hline$K_{k+1}$ & Kalman filter gain matrix \\
\hline$k$ & data frame index \\
\hline$L$ & length of pneumatic tubing \\
\hline$N$ & number of data frames available \\
\hline$O$ & output from pressure sensor \\
\hline$\hat{O}$ & deconvolution output estimate \\
\hline$P_{a}$ & initial local pressure \\
\hline$P_{k+1 / k}$ & prediction error covariance matrix \\
\hline$P_{k+1 / k+1}$ & filter error covariance matrix \\
\hline$P_{k+1 / N}$ & smoother error covariance matrix \\
\hline
\end{tabular}




$\begin{array}{ll}P_{L} & \text { downstream pressure response } \\ P_{0} & \text { prescribed input pressure function } \\ P(x, t) & \text { pressure function } \\ Q_{k+1} & \text { state noise covariance matrix } \\ Q_{U_{k+1}} & \begin{array}{l}\text { prescribed input function error variance } \\ R\end{array} \\ & \begin{array}{l}\text { acoustic resistance of pneumatic } \\ \text { configuration }\end{array} \\ R_{k+1} & \text { residual error vector } \\ t & \text { time coordinate } \\ U_{k+1} & \text { input vector } \\ V & \text { enclosed transducer volume } \\ V & \text { effective sensor volume, } V+\frac{A_{f} L}{2} \\ x & \text { spatial coordinate } \\ X & \text { state vector } \\ Z & \text { observation vector } \\ \tilde{Z} & \text { filter response residual }\end{array}$

Greek

$\Gamma_{k+1} \quad$ prescribed input vector

$\Delta_{k+1} \quad$ recursive residual error matrix

$\delta_{k+1} \quad$ input error vector

$\Delta t \quad$ discretization time interval

$\mu \quad$ dynamic viscosity

$\xi \quad$ damping ratio of reduced-order model

$\rho_{0} \quad$ linearization density

$\Phi \quad$ state equation transition matrix

$\omega_{n} \quad$ natural radian frequency of reduced-order model

\section{Introduction}

Recent advances in aircraft performance and maneuver capability have complicated the problem of flight control augmentation. Increasingly, aircraft system designs use acrodynamic parameters derived from pneumatic measurements as control system feedbacks. To be used as control feedbacks, pneumatically derived data must be measured with accuracy and dynamic fidelity. This has been difficult because of pressure distortion caused by frictional attenuation and pneumatic resonance within the sensing system. Typically, most distortion occurs within the pneumatic tubing used to transmit pressure impulses from the aircraft's surface to the measurement transducer.

To avoid pneumatic distortion, experiment designers mount the pressure sensor at the aircraft's surface, called in situ mounting. In some cases this technique is a viable solution. In most cases, as when many pressures must be measured in a small surface area or when pressure is sensed in an extemal environment, pressure sensor installations require pneumatic tubing from the aircraft's surface to the pressure transducer.

New non-intrusive pressure sensing technologies, such as piezo-film or optical techniques such as laser/Doppler, need to be developed. These tochnologies, however, are still being developed and will not be reliably proven for at least a decade. Additionally, these technologies are expensive. A research effort was initiatcd at the National Aeronautics and Space Administration Ames Research Center Dryden Flight Research Facility, supported by the University of California, Los Angeles, to develop a technique for measuring unsteady pressure data with conventional pressure sensing technology. Preliminary results of this research have been published in Refs. 1-3. This paper presents further results.

A pneumatic distortion model, developed in the early stages of this research, ${ }^{2,3}$ will be reduced to a low-order, state-variable model which retains most of the dynamic characteristics of the full model. The reduced-order model will be coupled with standard results from minimum variance estimation theory to develop an algorithm to compensate for the effects of pneumatic distortion. Both postflight and real-time algorithms are developed. Algorithm characteristics are evaluated using simulation and flight data.

\section{Background}

For most flight test applications pressure sensing devices are located remotely from the aircraft surface, usually within the internal cavity of the aircraft. A connecting length of pneumatic tubing transmits pressure changes at the aircraft's surface to the pressure transducer. Pressure variations at the surface propagate as waves from the upstream end through the pneumatic tubing to the transducer. The wave propogation is damped by frictional attenuation along the walls of the pneumatic tubing. This damping causes spectral attenuation of the pressure response and produces both a magnitude attenuation and a phase lag. When the wave arrives at the downstream (transducer) end of the pneumatic tubing, it is reflected back up the tube. Depending on the frequency of the incoming waves and the length of the pneumatic tubing, the reflected waves either cancel or reinforce incoming pressure waves. If the waves carcel, further spectral attenuation occurs; if the waves reinforce, the power of the incoming wave is amplified and acoustic resonance occurs. Spectral distortion will not occur in zero volume configurations (with in situ mounting) only. This paper will discuss modeling and compensating for the effects of internal acoustic dynamics of the pressure sensor. External effects, such as orifice disturbance of the surface flow, will not be modeled.

A large body of information on the effects of pneumatic distortion is available. Early analyses modeled the pneumatic tubing dynamics for specialized inputs ${ }^{4-9}$ assuming either highly damped or undamped measurement configurations. While accurately predicting pressure responses for 
defined types of inputs, these methods could not predict the behavior of pneumatic systems subjected to arbitrary pressure inputs.

A complex wave model which evaluates the sensor output for arbitrary inputs was developed in Refs. 2 and 3. The model, based on the unsteady Navier-Stokes equations of momentum and continuity, was verified by comparisons to special case analytical solutions, laboratory data, and flight data. The results of this verification indicate that predictions of the wave model are extremely accuratc. References 1-3 give detailed descriptions of the tests used to verify the model.

Because the wave model is complex, it cannot be easily inverted to develop a compensation routine which predicts the sensor input (the surface pressure) from the sensor output (the measured pressure). Thus the wave model must be reduced to a simpler, lower order model retaining most of the dynamic characteristics of the full wave model. The lower order model is more amenable to analytical manipulation and will be used to derive the compensation algorithms.

\section{Modeling and Analysis}

This section presents the idealized configuration to be analyzed first. Next, the full wave model of Refs. 2 and 3 will be approximated by a simple second-order filter which retains most of the dynamic characteristics of the complete model. The reduced-order model will be written in statevariable form and its frequency response will be compared with the full wave model's.

\section{Idealized Pressure Sensor Geometry and Conditions}

The pressure sensor configuration (Fig. 1) is modeled as a straight, cylindrical tube with an axisymmetric volume attached at the downstream end. The tube is of constant diameter $(D)$ and length $(L)$. The attached volume $(V)$, representing the internal volume of the pressure transducer, is also constant. A longitudinal coordinate $(x)$ starts at the upstream end of the tube, and a time coordinate $(t)$ starts at some initial time.

A small pressure pulse is introduced at the upstream end of the pneumatic tubing and propagates down the tube as a longitudinal wave. The flow velocity is approximated by the radial average, and as a result the pressure within the sensor is modeled as a function of $x$ and $t$ only. Since the sensor is closed at one end, flow velocities are small and air density within the sensor has only small variations. Since the flow velocities are small, temperature within the sensor is nearly constant.

\section{Governing Equations}

The governing equations of the full wave model are derived in Ref. 3. This model, a hyperbolic boundary value problem (BVP), defines the input pressure and solves for the downstream response. The basic dynamics equation is

$$
\frac{\partial^{2} P(x, t)}{\partial t^{2}}+\frac{R}{\rho_{0}} \frac{\partial P(x, t)}{\partial t}=c^{2} \frac{\partial^{2} P(x, t)}{\partial x^{2}}
$$

where $\rho_{0}$ is the initial density, $P(x, t)$ is the local pressure within the tubing, and $c$ is the local sonic velocity. The parameter $R$ is the acoustical resistance which for laminar flow is given by

$$
R=\frac{32 \mu}{D^{2}}
$$

where $\mu$ is the local dynamic viscosity. The system is assumed to be at rest initially; thus the defined initial conditions are

$$
P(x, 0)=P_{a}, \text { and } \frac{\partial P(x, t)}{\left.\partial t \quad\right|_{t=0}}=0
$$

where $P_{a}$ is an arbitrary local starting pressure and the defincd pressure function at the upstream boundary is

$$
P(0, t)=P_{0}(t)
$$

Srisfying momentum and continuity at the downstream boundary

$$
\frac{d^{2} P(L, t)}{d t^{2}}+\frac{R}{\rho_{0}} \frac{d P(L, t)}{d t}+\left.\frac{A_{c} c^{2}}{V} \frac{\partial P(x, t)}{\partial x}\right|_{x=L}=0
$$

where $P(L, t)$ is the sensor response at the pressure transducer. Equations (1) to (5) represent a completely and properly posed BVP. The existence and uniqueness of its solution is demonstrated in Ref. 3. Numerical solutions are obtained using implicit differencing in which the pressure function is approximated by a series of discrete gridpoints, with partial derivative operators approximated by finite differences. The resulting nonsymmetric tridiagonal system is solved using traditional elimination techniques. ${ }^{10}$

\section{Reducing the Wave Model to a Second-Order Equation}

Analyses of numerical solutions to the wave model indicate that for small-amplitude pressure inputs to low-volume sensors, the pressure gradient at the downstream boundary cuin be approximated by the spatial average of the gradient within the pneumatic tubing without substantially altering the dynamic characteristics of the model. If this approximation is performed and the BVP is integrated twice with respect to position, the wave model reduces to a second-order equation of the form ${ }^{3,7}$

$$
\frac{d^{2} P_{L}(t)}{d t^{2}}+2 \xi \omega_{n} \frac{d P_{L}(t)}{d t}+\omega_{n}^{2} P_{L}(t)=\omega_{n}^{2} P_{0}(t)
$$

where $\omega_{n}$ is the natural frequency, and $\xi$ is the equivalent damping ratio. For this model

$$
\begin{aligned}
\omega_{n}^{2} & =\frac{c^{2} A_{c}}{L V_{e}} \\
2 \xi \omega_{n} & =\frac{R}{\rho_{0}}
\end{aligned}
$$




$$
V_{e}=V+\frac{L A_{c}}{2}
$$

If equation (6) is integrated using a backward zero-order hold, ${ }^{11}$ the reduced-order model can be written in statevariable form as the matrix equation

$$
\begin{aligned}
\left(\begin{array}{c}
P_{L_{k}} \\
P_{L_{k+1}}
\end{array}\right)= & \left(\begin{array}{cc}
0 & 1 \\
\frac{-1}{1+2 \xi \omega_{n} \Delta t+\left(\omega_{n} \Delta t\right)^{2}} & \frac{2\left(1+\xi \omega_{n} \Delta t\right)}{1+2 \xi \omega_{n} \Delta t+\left(\omega_{n} \Delta t\right)^{2}}
\end{array}\right) \\
& \times\left(\begin{array}{c}
P_{L_{k-1}} \\
P_{L_{k}}
\end{array}\right) \\
& +\left(\begin{array}{c}
0 \\
\frac{\left(\omega_{n} \Delta t\right)^{2}}{1+2 \xi \omega_{n} \Delta t+\left(\omega_{n} \Delta t\right)^{2}}
\end{array}\right) P_{0_{k+1}}
\end{aligned}
$$

As mentioned earlier, equation (7) retains most of the frequency and time response characteristics of the original wave model up through the first wave harmonic. This is shown by comparing the analytical frequency responses for the full and reduced-order models. Figure 2 shows frequency response results for an overdamped sensor with pneumatic tubing $8 \mathrm{ft}$ long and 0.06 -in. diameter and a negligible volume transducer. The operating altitude is $40,000 \mathrm{ft}$. The equivalent damping ratio of the reduced-order model is 1.93 , and the natural frequency is $26.75 \mathrm{~Hz}$. Figure 3 shows frequency response comparisons for a lightly damped sensor with the same configuration operating at an altitude of $20,000 \mathrm{ft}$. For this configuration, the equivalent damping ratio of the reduced-order system is 0.25 and the natural frequency is $108.93 \mathrm{~Hz}$.

For both figures the comparisons are good up to the natural frequency of the reduced-order model. Beyond this both the magnitude and phase angle comparisons begin to diverge. The reason for this divergence is clear; the secondorder approximation does not allow for higher order wave harmonics, while the full wave model does. Thus at frequencies beyond the first harmonic, the reduced-order model may not be acceptable and results of the compensation routine in the next section will probably not be accurate beyond the first wave harmonic. For analysis beyond the first wave harmonic a higher order model which allows for additional wave harmonics can be used. The model parameters can be matched to the full wave model using system identification or resonance peak matching techniques.

\section{Deriving the Compensation Algorithm}

The compensation algorithm will be developed in this section. The objective is to develop an algorithm to infer the external pressure input to the measurement system based on observations of the pressure response at the transducer. In general, the technique of infering a system input when given knowledge of the system structure and a measurement of the system response is called deconvolution. For this application, this is a difficult task.

If equation (7) is solved for $P_{0_{k+1}}$ in terms of $P_{L_{k-1}}$, $P_{L_{k}}$, and $P_{L_{k+1}}$, the resulting equation is numerically illconditioned; small changes in $P_{L_{k}}$ produce large changes in $P_{0_{k+1}}$. As a result, noise in the pressure measurements at the transducer end of the sensor is over amplified by the numerical instability of the computation and overwhelms any estimate of the input pressure. The measurement noise must be identified and controlled as a part of an estimation loop. The algorithm that performs the error control function, presented in this section, will be used to infer the external pressure input to the system based on observations of the pressure response at the transducer.

First, a general deconvolution algorithm as developed in Ref. 3 will be presented. The technique, developed using the techniques of minimum variance estimation theory, is similar to results presented in Refs. 12-14. Both postflight and real-time versions of the algorithm will be presented. After the general algorithms are presented, they will be applied to the pressure compensation problem.

\section{Minimum Variance Deconvolution Algorithm}

The deconvolution algorithm defines an input to the statevariable model and identifies the residual between the prescribed input and the actual input using minimum variance estimation techniques. All notational conventions in this paper follow the conventions of Ref. 15 . The assumed form of the model is

$$
\begin{aligned}
X_{k+1} & =\Phi X_{k}+B\left[\Gamma_{k+1}+\delta_{k+1}\right] \\
Z_{k+1} & =C X_{k+1}+g_{k+1}
\end{aligned}
$$

where,

$$
U_{k+1}=\Gamma_{k+1}+\delta_{k+1}
$$

In equation (10) $U_{k+1}$ is the actual input to the system, $\Gamma_{k+1}$ is the defined (nonrandom) input to the system model, and $\delta_{k+1}$ is a random process which models the residual between the actual and prescribed inputs. The covariance of $\delta_{k+1}$ is defined as

$$
\operatorname{COV}\left[\delta_{k+1}\right]=Q_{k+1}
$$

and is assumed to be known.

Measurements of the state vector $\left(Z_{k+1}\right)$ are observed in the presence of noise. The measurement noise covariance is given as

$$
\operatorname{COV}\left[g_{k+1}\right]=G_{k+1}
$$

and is assumed to be known.

For notational convenience, the state transition matrix $(\Phi)$ and the input and measurement geometry matrixes $(B$ and $C$ ) are written as constants. 
Two complete estimation algorithms will be presented. The first, intended for postllight data reconstruction, is implemented as a two-pass forward filter, backward smoother. As developed in Ref. 3, the posuflight smoothing algorithm relies on the use of all available data measurements, both past and future. The algorithm, a hybrid of the Kalman filter and the Rauch smoother, ${ }^{15}$ uses information in the Kalman filter innovations to identify differenees between the defined input and the true input during the reverse pass of the algorithm. The second algorithm, intended for real-time operation, will be derived from the smoothing algorithm and implemented as a time-recursive filter. This algorithm does not use information from future observations of the system response.

Postflight Smoothing Algorithm. Assuming that observations of the system response are available for data frames $k=0,1, \ldots, N$, the following scquence of computations are required for the smoothing algorithm:

Kalman filter loop, performed for $k=0,1, \ldots, N$

$$
\begin{aligned}
\hat{X}_{k+1 / k} & =\Phi \hat{X}_{k / k}+B \Gamma_{k+1} \\
P_{k+1 / k} & =\Phi P_{k / k} \Phi^{\mathrm{T}}+B Q_{k+1} B^{\mathrm{T}} \\
\tilde{Z}_{k+1 / k} & =Z_{k+1}-C \hat{X}_{k+1 / k} \\
K_{k+1} & =P_{k+1 / k} C^{\mathrm{T}}\left[C P_{k+1 / k} C^{\mathrm{T}}+G_{k+1}\right]^{-1} \\
\hat{X}_{k+1 / k+1} & =\hat{X}_{k+1 / k}+K_{k+1} \tilde{Z}_{k+1 / k} \\
P_{k+1 / k+1} & =\left[I-K_{k+1} C\right] P_{k+1 / k}
\end{aligned}
$$

where $P_{k+1 / k}$ is the predicted filter error covariance, $P_{k+1 / k+1}$ is the adjusted filter error covariance based on the current observation, $K_{k+1}$ is the Kalman filter gain matrix, and $\tilde{Z}_{k+1 / k}$ is the filter innovation. The matrixes $B Q_{k+1} B^{\mathrm{T}}$ and $G_{k+1}$ are the assumed covariances for the input and measurement noise processes. The vectors $\hat{X}_{k+1 / k}$ and $\hat{X}_{k+1 / k+1}$ are the predicted and corrected estimates of the filter state vector. Resulting estimates for the state vector, the crror covariances, and the innovations process must be stored for each recursion, $k=0,1, \ldots, N$.

At this point the input residual estimation loop is performed backwards for $k=N, N-1, \ldots, 0$, using stored data

$$
\begin{aligned}
R_{k / N} & \left.=\Delta_{k}^{\mathrm{T}} R_{k+1 / N}+C^{\mathrm{T}} \mid C P_{k / k-1} C^{\mathrm{T}}+G_{k}\right]^{-1} \tilde{Z}_{k / k-1} \\
\hat{\delta}_{k / N} & =Q_{k} B^{\mathrm{T}} R_{k / N}
\end{aligned}
$$

where $R_{k / N}$ is the deconvolution residual vector, defined by

$$
P_{k / k-1} R_{k / N}=\hat{X}_{k / N}-\hat{X}_{k / k-1}
$$

and

$$
\Delta_{k}^{\mathbf{T}}=\left(I-K_{k} C\right)^{\mathrm{T}} \boldsymbol{\Phi}^{\mathrm{T}}=\left[\boldsymbol{\Phi}\left(I-K_{k} C\right)\right]^{\mathrm{T}}
$$

Finally, the input estimate is computed as

$$
\hat{U}_{k / N}=\Gamma_{k / N}+\hat{\delta}_{k / N}
$$

The final value for the residual vector, $R_{N+1 / N}$, is given by equation (11) with $k$ replaced by $N$

$$
R_{N+1 / N}=\left\{P_{N+1 / N}\right]^{-1}\left[\hat{X}_{N+1 / N}-\hat{X}_{N+1 / N}\right\}=0
$$

Real-Time Filtering Algorithm. The real-time algorithm relies only on past and present data to perform the estimation. Assuming that observations of the system response are available for data frames $k=0,1, \ldots, k+1$, the residual estimation is performed with $k+1$ replacing $N$

$$
\begin{aligned}
\hat{\delta}_{k+1 / k+1} & =Q_{k+1} B^{\mathrm{T}} R_{k+1 / k+1} \\
i \imath_{k+1 / k+1} & =\left[P_{k+1 / k}\right]^{-1}\left[\hat{X}_{k+1 / k+1}-\hat{X}_{k+1 / k}\right]
\end{aligned}
$$

therefore,

$$
\hat{\delta}_{k+1 / k+1}=Q_{k+1} B^{\mathrm{T}}\left[P_{k+1 / k}\right]^{-1}\left[\hat{X}_{k+1 / k+1}-\hat{X}_{k+1 / k}\right]
$$

and substituting in the Kalman filter

$$
\hat{\delta}_{k+1 / k+1}=Q_{k+1} B^{\mathrm{T}}\left[P_{k+1 / k}\right]^{-1} K_{k+1} \tilde{Z}_{k+1 / k}
$$

Applying the definition of the Kalman gain matrix

$$
\hat{\delta}_{k+1 / k+1}=Q_{k+1} B^{\mathrm{T}} C^{\mathrm{T}}\left[C P_{k+1} C^{\mathrm{T}}\right]^{-1} \bar{Z}_{k+1 / k}
$$

The input may be estimated as a part of the Kalman filtering loop according to the sequence of computations:

Kalman filter step performed for $k=0,1, \ldots, N$

$$
\begin{aligned}
\hat{X}_{k+1 / k} & =\Phi \hat{X}_{k / k}+B \Gamma_{k+1} \\
P_{k+1 / k} & =\Phi P_{k / k} \Phi^{\mathrm{T}}+B Q_{k+1} B^{\mathrm{T}} \\
\tilde{Z}_{k+1 / k} & =Z_{k+1}-C \hat{X}_{k+1 / k} \\
K_{k+1} & =P_{k+1 / k} C^{\mathrm{T}}\left[C P_{k+1 / k} C^{\mathrm{T}}+G_{k+1}\right]^{-1} \\
\hat{X}_{k+1 / k+1} & =\hat{X}_{k+1 / k}+K_{k+1} \tilde{Z}_{k+1 / k} \\
P_{k+1 / k+1} & =\left[I-K_{k+1} C\right] P_{k+1 / k}
\end{aligned}
$$

Residual estimation step

$$
\hat{\delta}_{k+1 / k+1}=Q_{k+1} B^{\mathrm{T}} C^{\mathrm{T}}\left[C P_{k+1} C^{\mathrm{T}}\right]^{-1} \tilde{Z}_{k+1 / k}
$$

and

$$
\hat{U}_{k+1 / k+1}=\Gamma_{k+1 / k+1}+\hat{\delta}_{k+1 / k+1}
$$

\section{Applying Minimum Variance Deconvolution Aigorithms to the Pressure Compensation Problem}

The above algorithms will now be applied to the pneumatic distortion problem. Equation (7) may be written as the first-order matrix difference equation

$$
X_{k+1}=\Phi X_{k}+B U_{k+1}
$$

If the downstream pressure is observed in the presence of noise, then

$$
Z_{k+1}=X_{k+1}+g_{k+1}
$$

If the substitution

$$
U_{k+1}=\Gamma_{k+1}+\delta_{k+1}
$$


is performed, equations (12) and (13) are the same form as the assumed deconvolution model, equations (8) and (9), and the deconvolution algorithms may be directly applied.

The input function $\left(\Gamma_{k+1}\right)$ is determined by solving cquation (7) for the input pressure in terms of the downstream pressures, and evaluating the result using state estimates from previous time recursions of the filter loop. Amplified noise in $\Gamma_{k+1}$ is estimated and controlled by the deconvolution algorithm through judicious selection of the state and measurement noise covariance matrixes.

The state noise covariance matrix is derived from equation (7) as

$$
B Q_{k+1} B^{\mathrm{T}}=\left(\begin{array}{cc}
0 & 0 \\
0 & Q_{U_{k+1}}\left[\frac{\left(\omega_{n} \Delta t\right)^{2}}{1+2 \xi \omega_{n} \Delta t+\left(\omega_{n} \Delta t\right)^{2}}\right]^{2}
\end{array}\right)
$$

where $Q_{U_{k+1}}$ is the variance in the residual $\delta_{k+1}$. Similarly, assuming that the measurement noise is white, the measurement noise covariance matrix is

$$
G_{k+1}=\left(\begin{array}{cc}
G(1)_{k+1} & 0 \\
0 & G(2)_{k+1}
\end{array}\right)
$$

where $G(1)_{k+1}$ is the error variance of the measured downstream pressure at data frame $k$ and $G(2)_{k+1}$ is the error variance in the measured downstream pressure at data frame $k+1$. For stationary measurement processes

$$
G(1)_{k+1}=G(2)_{k+1} \equiv G
$$

\section{Results of Deconvolution Analysis}

Selected results of the minimum variance deconvolution analysis will now be presented. Filtering tuning will be demonstrated using simulated data. Next the deconvolution algorithms will be applied to flight data. Both the postflight smoothing and real-time filtering algorithms will be analyzed and compared with reference pressure data oblained from flight experiments.

\section{Filter Tuning}

Selecting proper assumed values of the error covariance matrixes is critical. This practice, referred to as "filter tuning," is typical of minimum variance estimators. The appropriate values for the covariance matrixes are usually unique to each configuration. Proper tuning of the deconvolution algorithms will be shown using simulated data generated from numerical solutions of the full wave model. Simulated data is used because the actual measurement and input error covariances can be predefined. The simulated data were generated using the full wave model assuming the configuration associated with the overdamped case of Fig. 2; a sensor configuration with pneumatic tubing $8 \mathrm{ft}$ long, and of 0.06 -in. diameter, a transducer with negligible volume, and operating at an altitude of $40,000 \mathrm{ft}$. For this configuration and altitude, the equivalent damping ratio of the reducedorder system is 1.93; the natural frequency is $26.75 \mathrm{~Hz}$. Simulated zero-mean white noise with a variance of $0.1\left(\frac{\mathrm{lb} \cdot \mathrm{f}}{\mathrm{f}^{2}}\right)^{2}$ was superimposed on the output time history; simulated zero-mean white noise with a variance of $1.0\left(\frac{\mathrm{lb}-f}{\mathrm{ft}^{2}}\right)^{2}$ was superimposed on the input time history. The input and output noise were uncorrelated.

The minimum variance deconvolution analysis was simulated using the postfight smoothing algorithm. Figure 4 shows the simulation process. A defined input is superimposed with additive noise and the result $(I)$ is input to the wave model. The output from the wave model is superimposed with additive noise to give a pseudomeasurement $(O)$ time history. Using values of the natural frequency and the equivalent damping ratio provided by the wave model and prescribed values for the input and observation covariances, the pseudomeasurement is used to perform the deconvolution. The resulting deconvolution estimates of the model input and response are indicated by the symbols $\hat{I}$ and $\hat{O}$.

Figures 5 and 6 show the effects of selected values of $G$ on the estimates of $U$. In these figures, the value of $Q U$ was fixed at the appropriate values of 1.0, while the value for $G$ was varied. Recall that the actual measurement error variance is $0.1\left(\frac{\mathrm{b} \cdot \mathrm{f}}{\mathrm{ft}^{2}}\right)^{2}$. Figure 5 shows the deconvolution results for $G=10$, a value which is much too large. Three time history graphs are shown. Time history overplots of the measured downstream pressure response $(O)$ and the smoother estimate of the input pressure $(\hat{I})$ are shown in the bottom graph. Time history overplots of $O$ and the estimated response $(O)$ are shown in the middle graph, and time history overplots of the actual input $(I)$ and $\hat{I}$ are shown in the top graph. Comparisons are poor. This set of weights does not allow the estimation algorithm to extract enough information from the measured pressure data; resulting estimates are overly smoothed.

The above situation cannot be improved by arbitrarily lowering the value for $G$. Figure 6 shows similar time history comparisons for a value of $G$ which is much too small, 0.01 . The end result is a virtually perfect match between the measured and predicted response. As discussed earlier, this results in over-amplification of the measurement noise and the estimate is overwhelmed. The selected value for $G$ did not allow the deconvolution algorithm to identify and control noise in the measured response. The filter is essentially trying to perform an open-loop deconvolution of the state equation.

Figure 7 shows the effect of selected values of $Q_{U}$ on the estimation of $U$. The value of $G$ was fixed at the appropriate value of 0.10 , while the value for $Q_{U}$ was set at 10 . The actual value for $Q_{U}$ is 1.0. The resulting estimate contains extraneous harmonics that are not present in the original input signal caused by overamplification of mid-range frequencics. The dynamic constraints of the reduced-order model have not been enforced strongly enough. 
The appropriate level of smoothing and compensation is shown in Fig. 8. Here the proper values for the mcasurement and input error covariances are used. In this case the estimated input matches the actual input well, and much of the superimposed noise has been removed.

\section{Application to Flight Data}

The deconvolution algorithms will now be applied to flight data from the experiments described in Refs. 1-3. The flight test configuration mounted on the wing of a highperformance aircraft (Fig. 9) consisted of a set of static test orficies connected by pneumatic tubing to a single clectronically scanned pressure module (ESP), and a single in situ mounted pressure sensor. The test matrix had ports of 0.02 , 0.04 , and 0.06 in. in diameter and allowed pneumatic tubing sections to be interchangeable. Reference pressure data were provided by a very low volume piezioclectric wafer pressure transducer glued to the aircraft skin adjacent to the test matrix. Since the in situ transducer was mounted on the aircraft skin, it was exposed to temperatures considerably lower than the recommended normal operating range. This caused the direct current (DC) output to drift off the scale. To eliminate the drift, the reference sensor output was clectronically coupled with a third-order band-pass filter (1.16 to $101.7 \mathrm{~Hz}$ ) to remove the DC component. The DC component was restored to the reference data using the output from a pressure port connected to the ESP module by 6 in. of pneumatic tubing. The output from this port was lowpass filtered using a second-order digital filter with a rolloff frequency at $1.0 \mathrm{~Hz}$. The two filtered signals were merged using a complementary filter.

Pressure data obtained from the test matrix measurements were compensated using both the postllight smoothing and real-time filtering algorithms. The results will be compared to the reference data reconstructed using the complementary filter. The flight data deconvolution process (Fig. 10) is similar to the simulation process described carlicr. Two flight data cases will be presented; one for an overdamped configuration, and one for an underdamped configuration.

Deconvolution results for the overdamped flight maneuver obtained at $42,000 \mathrm{ft}$ altitude with a sensor configuration with $8 \mathrm{ft}$ of 0.06 -in.-diameter pneumatic tubing and negligible transducer volume are shown in Fig. 11 for the smoothing algorithm and Fig. 12 for the filtering algorithm. For this configuration and altitude, the equivalent damping ratio of the reduced-order system is 2.125 ; the natural frequency is $26.75 \mathrm{~Hz}$. Again, three time history plots are shown. The lower graph compares $\hat{I}$ with $O$. The middle graph compares $O$ and $\hat{O}$. The upper graph compares $I$ to $\hat{l}$. The comparisons are excellent for both the postflight smoothing and real-time filtering algorithms. The estimated input does not contain the high-frequency noise present in the reference data.

Figure 13 shows deconvolution results for an underdamped flight maneuver at $24,000 \mathrm{ft}$ altitude with a sen- sor configuration having $2 \mathrm{ft}$ of 0.06 -in.-diameter pneumatic tubing and negligible transducer volume for the smoothing algorithm. Figure 14 shows results for the filtering algorithm for the same configuration and altitude. For this configuration and altitude, the equivalent damping ratio of the reduced-order system is 0.279 ; the natural frequency is $107.18 \mathrm{~Hz}$. Both the smoothing and filtering algorithms work well. The smoothing algorithm removes nearly all of the resonant harmonic, approximately $100 \mathrm{~Hz}$, from the measured pressure response. The filtering algorithm removes a sizable portion of the resonance. Both algorithms account for phase lag well.

These results are typical of most flight maneuvers analyzed. Both the postlight smoothing and real-time filtering algorithms performed well. All tuning of the filter covariances was perform off-line using preflight information and arbitrary selection. No on-line adaptation of the covariances was performed.

\section{Summary and Concluding Remarks}

In this paper, a general numerical technique for obtaining unsteady pressure measurements using conventional pressure sensing technology was developed. A mathematical model, based on the Navier-Stokes equations of momentum and continuity, was reduced to a simple state-variable model. Frequency responses of the full and reduced-order models were shown.

Using a collection of standard results from minimum variance estimation theory, the reduced-order model was incorporated into a deconvolution algorithm which estimates the system input based on the system output. Both postfight smoothing and real-time filtering algorithms were developed. Overamplification of measurement error is controlled by careful selection of the elements of the algorithm state and measurement error covariance matrixes.

Performance characteristics were discussed. Filter tuning characterisitcs were demonstrated using simulated data. Applications of the techniques to flight data were presented. Both the postflight smoothing and real-time filtering algorithms performed well for overdamped and underdamped sensor configurations.

The results of this research are promising for the modeling and compensation techniques developed. These techniques provide a means of predicting and accounting for the effects of pneumatic distortion in pressure sensing devices. They also offer a reliable and cost effective means of measuring unsteady pressure data. These techniques will be useful until new pressure sensing technologies can be developed and proven.

\section{References}

${ }^{1}$ Curry, Robert E., and Gilyard, Glenn B., Flight Evaluation of a Pneumatic System for Unsteady Pressure Measurements Using Conventional Sensors, NASA TM-4131, 1989. 
${ }^{2}$ Whitmore, Stephen A., Formulation of a General Technique for Predicting Pneumatic Altenuation Errors in Airborne Pressure Sensing Devices, NASA TM-100430, 1988.

${ }^{3}$ Whitmore, Stephen A., Formulation and Verification of a Techique for Compensation of Pneumatic Attenuation Errors in Airborne Pressure Sensing Devices, Ph.D. Dissertation, University of Califormia, Los Angeles, University Microfilms International, 1989.

${ }^{4}$ Berg, H., Tijdeman, H., "Theoretical and Experimental Results for the Dynamic Response of Pressure Measuring Systems," NLR report F.238, 1965.

${ }^{5}$ Huston, W.B., The Accuracy of Airspeed Measurements and Flight Calibration Procedures, NACA Report 919. 1948.

${ }^{6}$ Iberall, A.S., Attenuation of Oscillatory Pressures in Instrument Lines, National Bureau of Standards Research Paper, RP 2115, July, 1950.

${ }^{7}$ Lamb, J.P., "The Influence of Geometry Parameters Upon Lag Error in Airbome Pressure Mcasurement Systems," WADC TR 57-351, Wright-Patterson AFB, Ohio, July 1957 .

${ }^{8}$ Schuder, C.B., and Binder, R.C., "The Response of Pneumatic Transmission Lines to Step Inputs," Journal of Basic Engineering, ASME Transactions, Dec. 1959.
${ }^{9}$ Stcphens, R.W.B., and Bate, A.E., Acoustics and Vibrational Physics, St. Martin Publishing Co., New York, 1966.

${ }^{10}$ Golub, Gene H., and Van Loan, Charles, F., Matrix Computations, John Hopkins University Press, Baltimore, 1983, pp. 100-108.

${ }^{11}$ Franklin, Gene F., and Powell, J. David, Digital Control of Dynamic Systems, Addison Wesley Publishing Co., Reading, Mass., 1980.

${ }^{12}$ Chi, Chong-Yung, "A Further Analysis of MinimumVariance Deconvolution Filter Performance," IEEE Transactions on Acoustics, Speech, and Signal Processing, Vol. ASSP-35, June 1987, pp. 888-889.

${ }^{13} \mathrm{Chi}$, Chong-Yung, and Mendel, J.M., "A Fast Approach to Identification Using Deconvolution," Institute of Electrical and Electronics Engineering Inc., Proceedings of the Conference on Decision and Control, Volume 3, 1983, pp. 1347-1352.

${ }^{14}$ Mendel, Jerry M., Optimal Seismic Deconvolution, An Estimation Based Approach. The Academic Press, New York, 1983.

${ }^{15}$ Mcditch, J.S., Stochastic Optimal Linear Estimation and Control, McGraw Hill Book Company, New York, 1969.

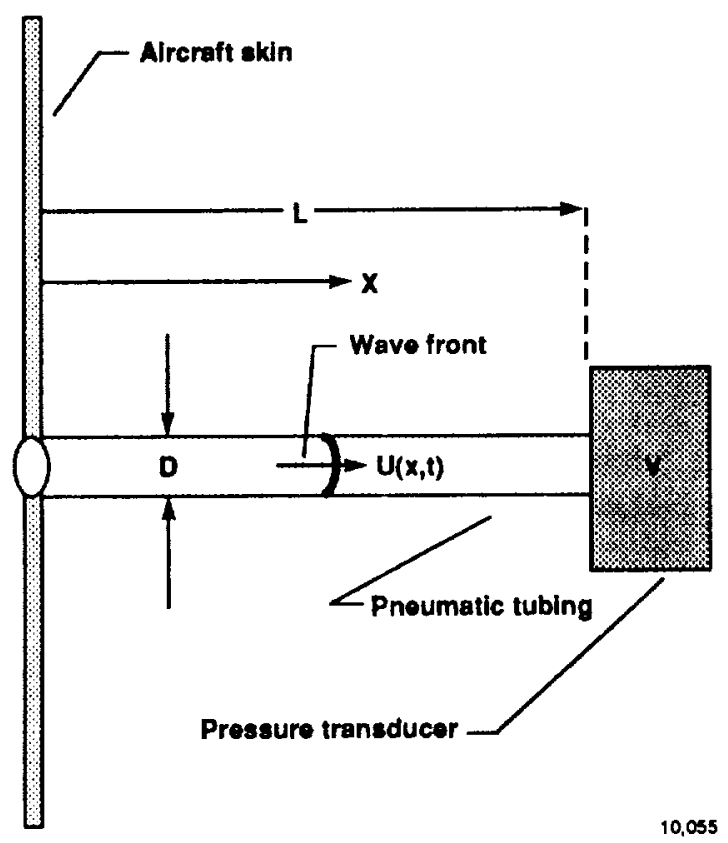

Fig. 1 Pressure sensing device. 

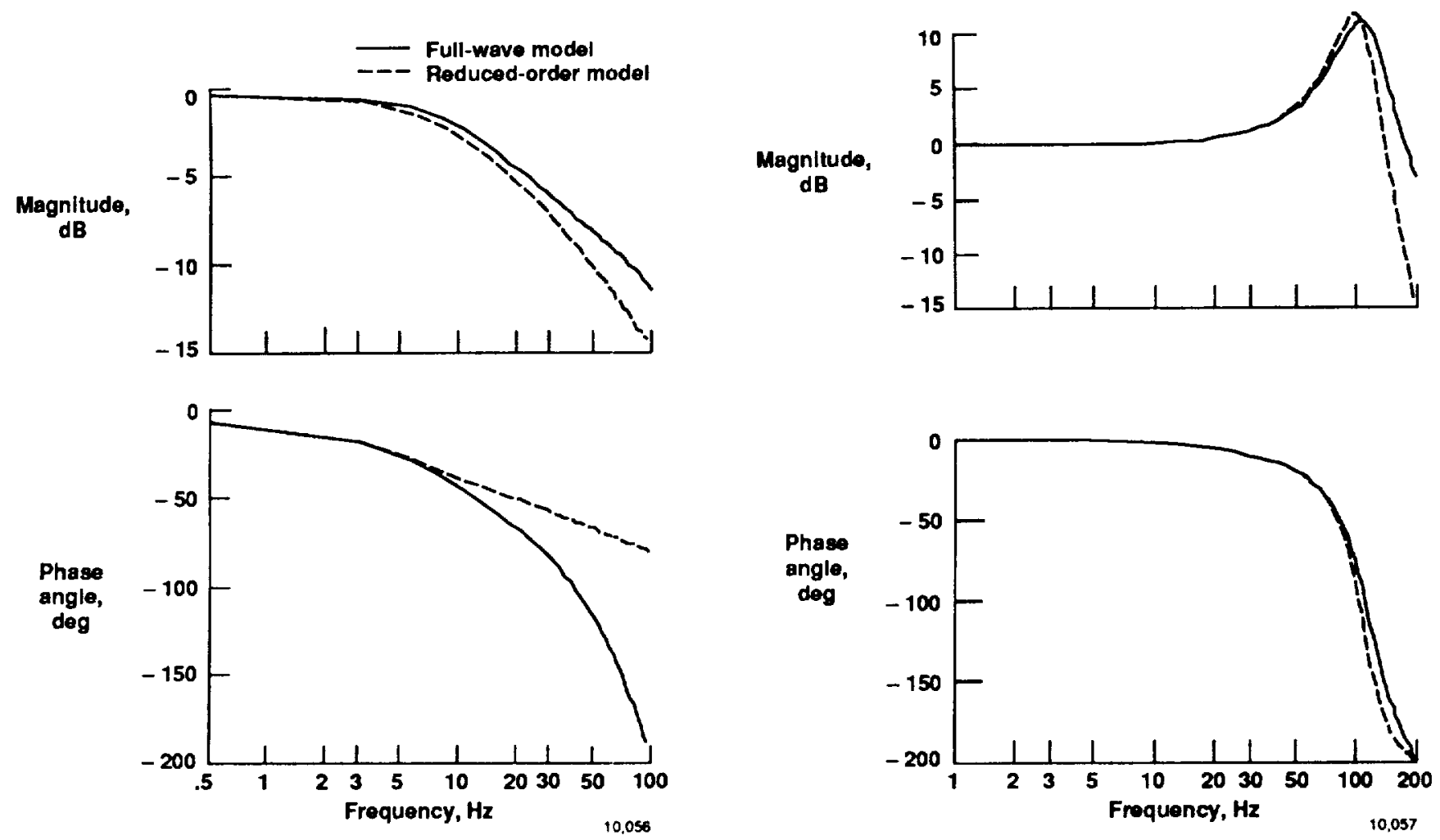

Fig. 2 Frequency response comparison of full and reduced-order models for overdamped sensor configuration.

Fig. 3 Frequency response comparison of full and reduced-order models for underdamped sensor configuration.

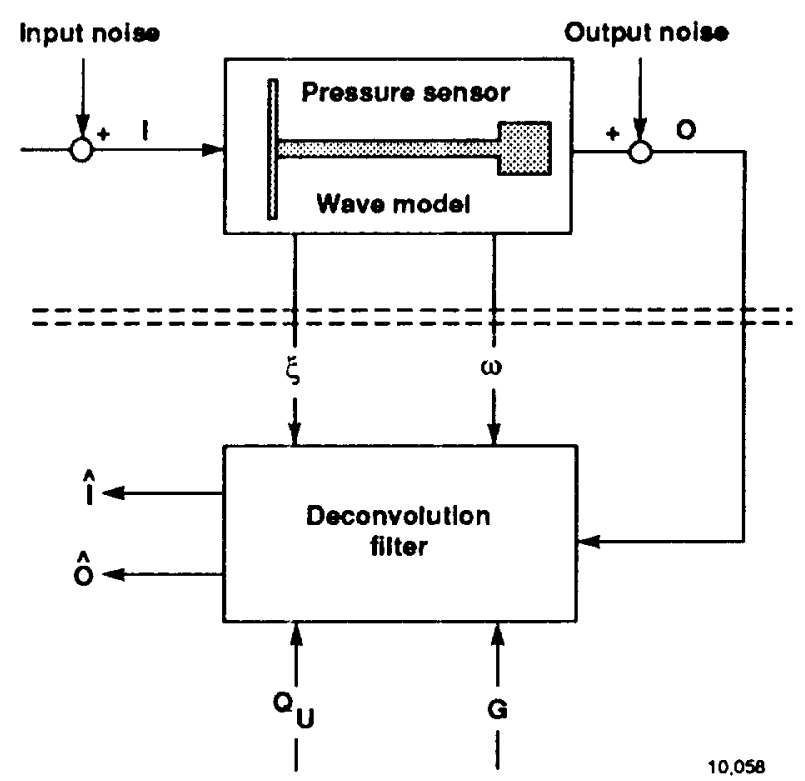

Fig. 4 Deconvolution simulation scheme. 

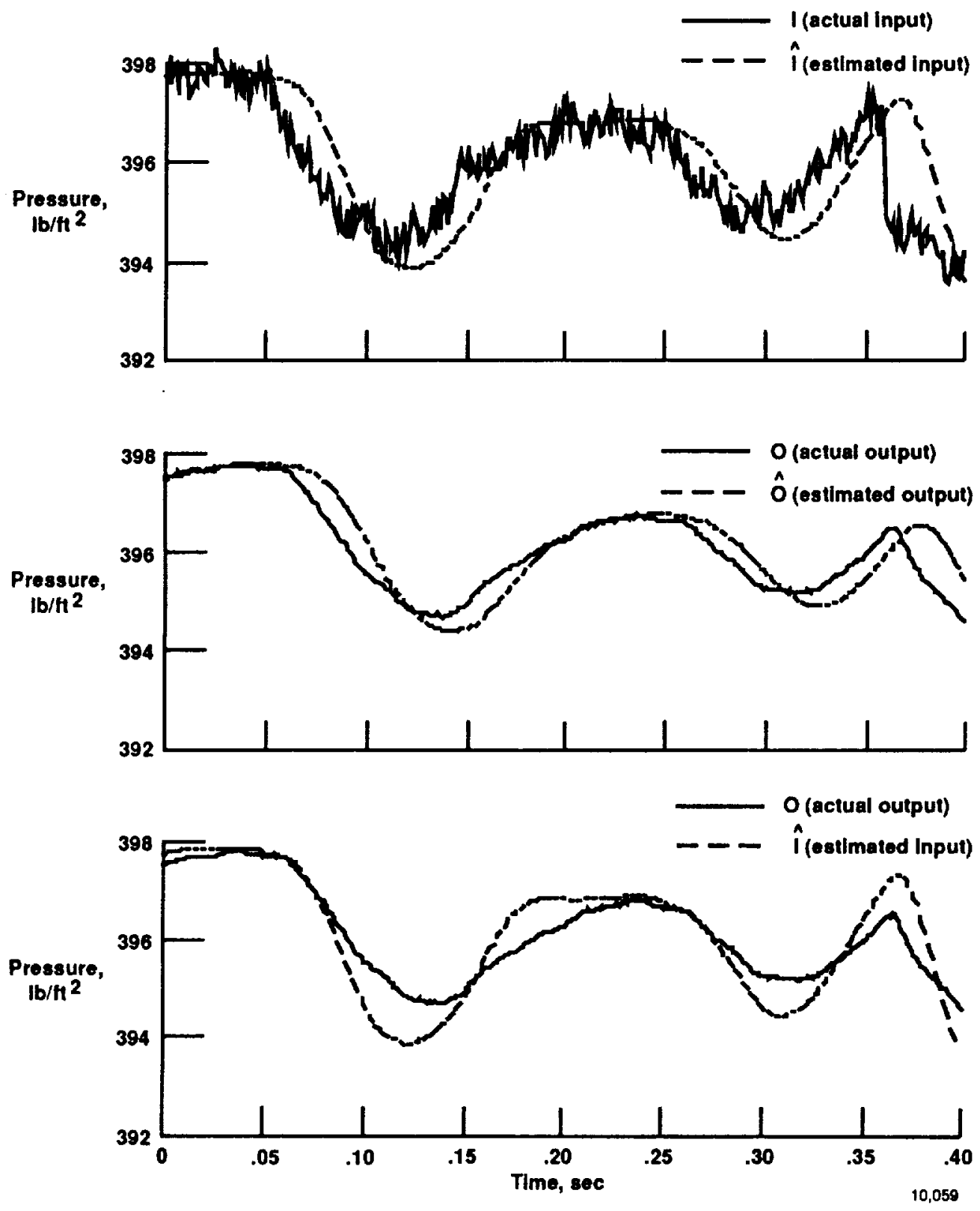

Fig. 5 Effect of improperly selected measurement error covariance, $Q_{U}=1.0, G=10.0$. 

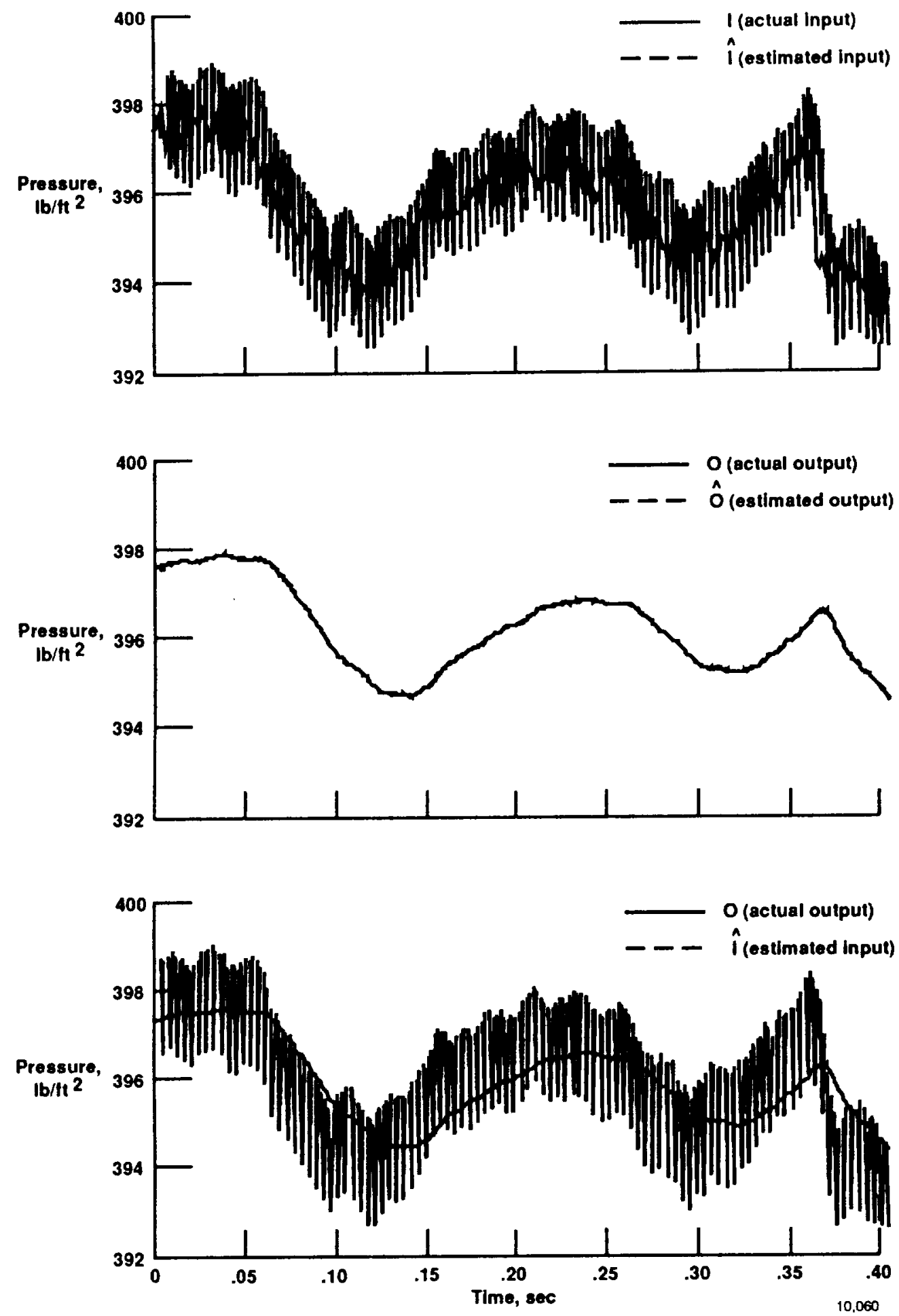

Fig. 6 Effect of improperly selected measurement crror covariance, $Q_{U}=1.0, G=0.01$. 

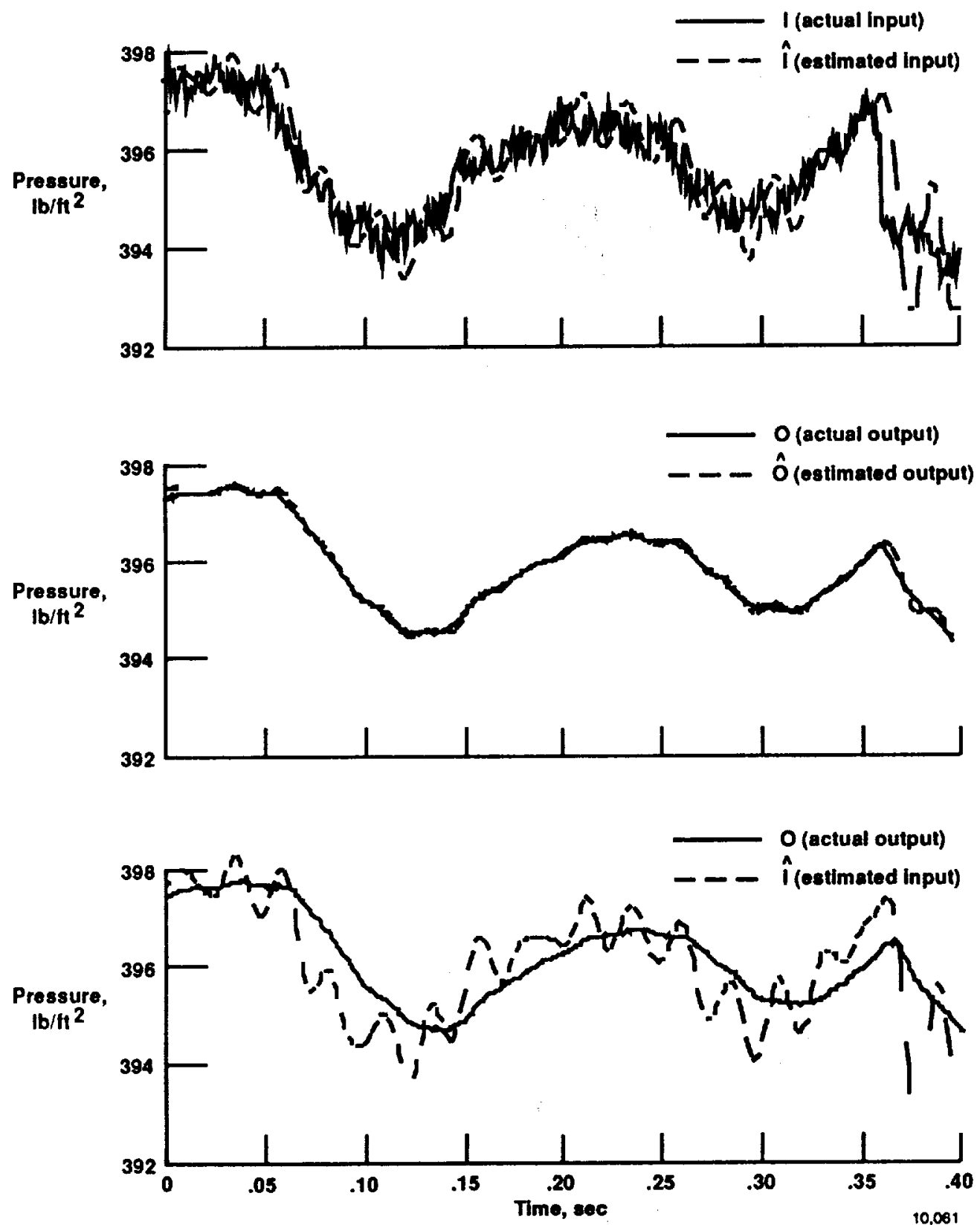

Fig. 7 Effect of improperly sclected input error covariance, $Q_{U}=10.0, G=0.1$. 

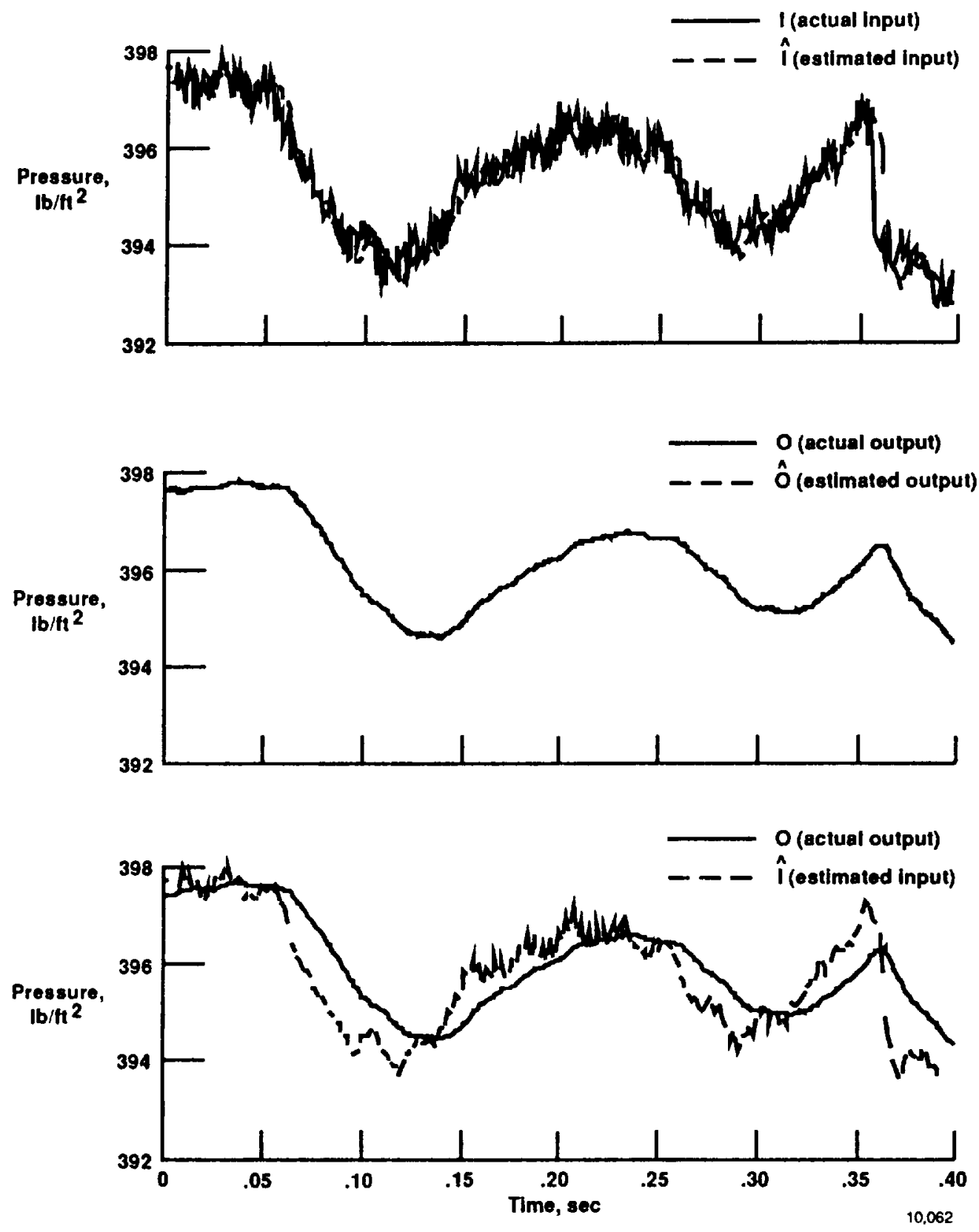

Fig. 8 Effect of properly selected input and measurement error covariance, $Q_{U}=1.0$, $G=0.1$. 


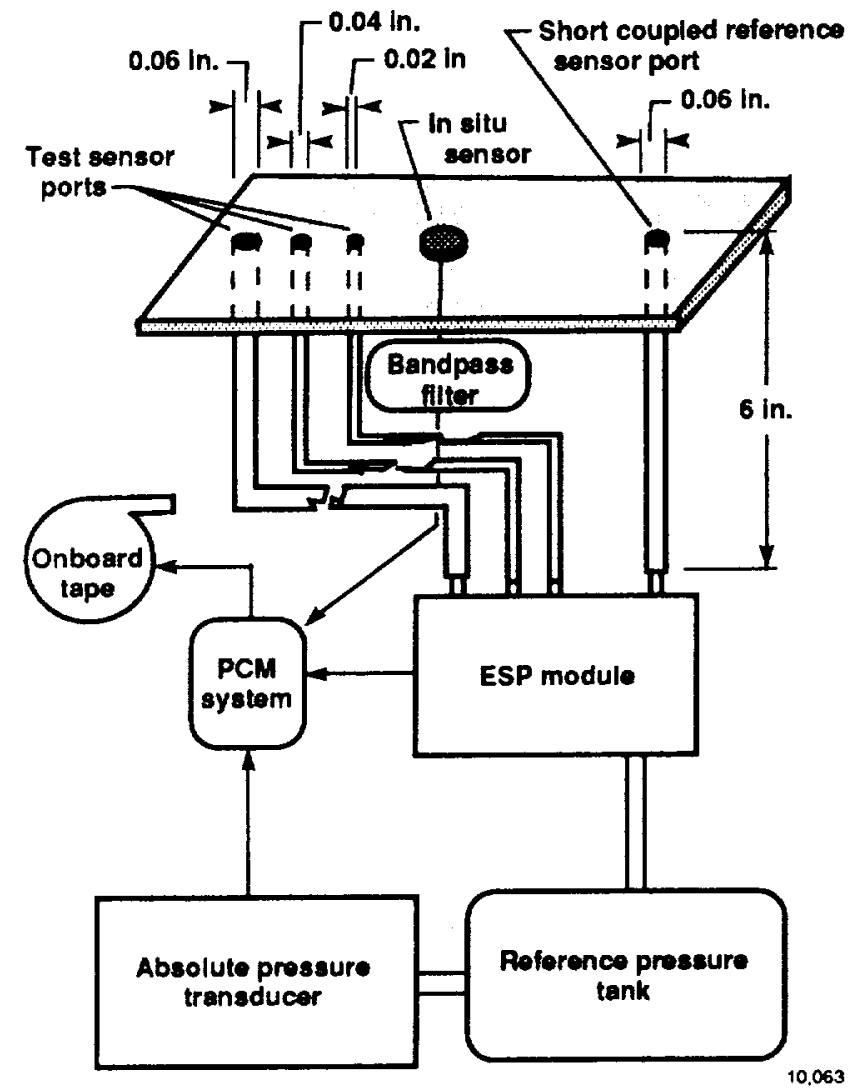

Fig. 9 Flight test experiment.

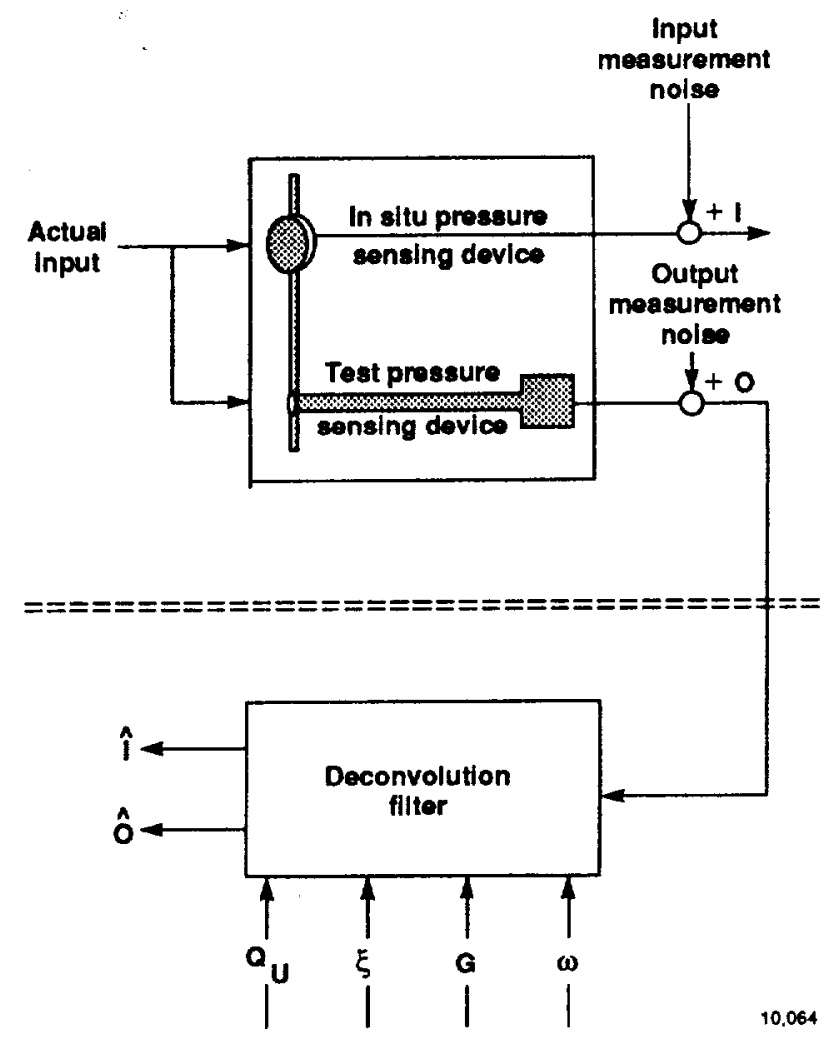

Fig. 10 Flight data deconvolution algorithm. 

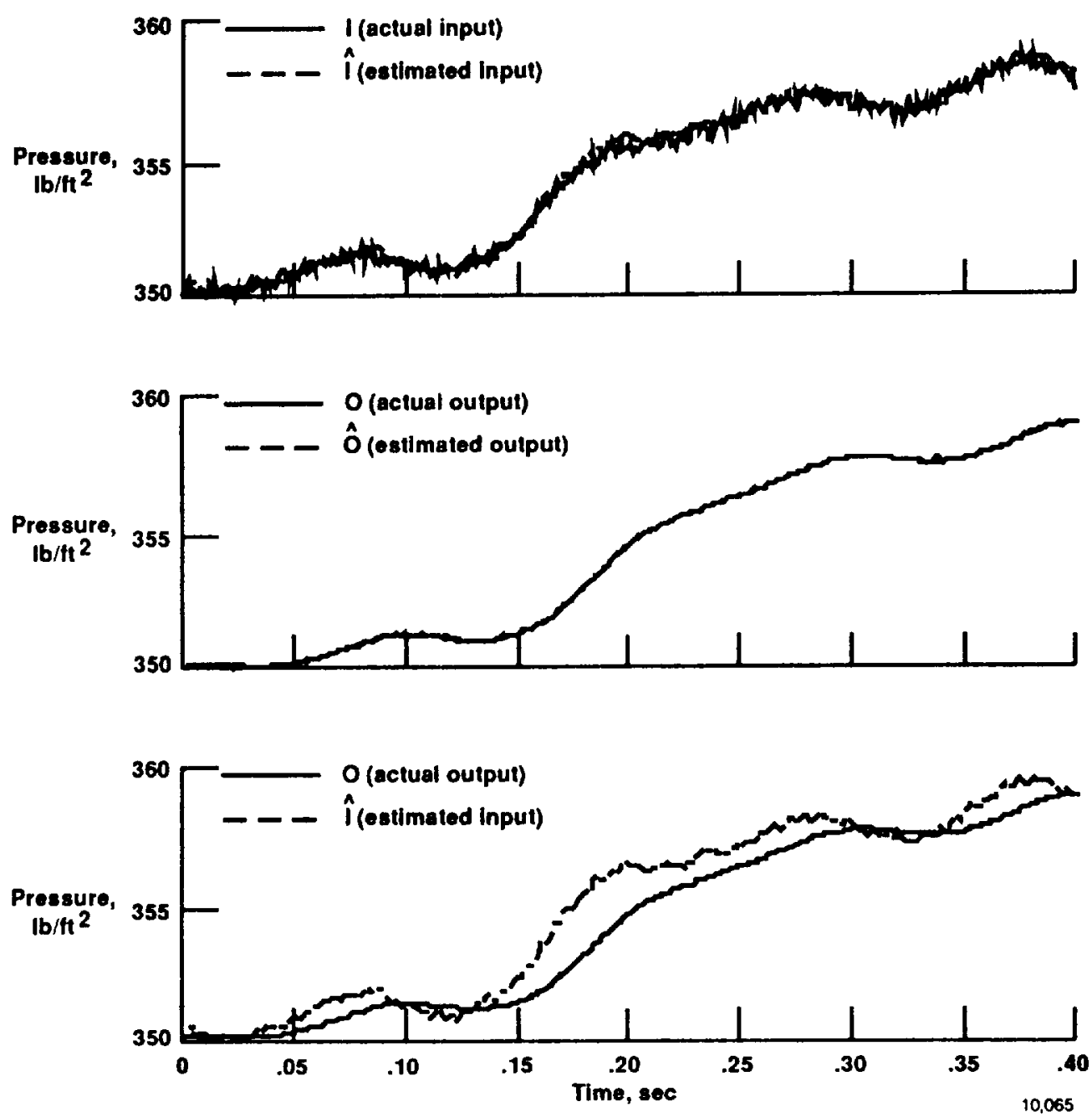

Fig. 11 Flight mancuver time history comparison for the posulight deconvolution algorithm output for an overdamped system. 

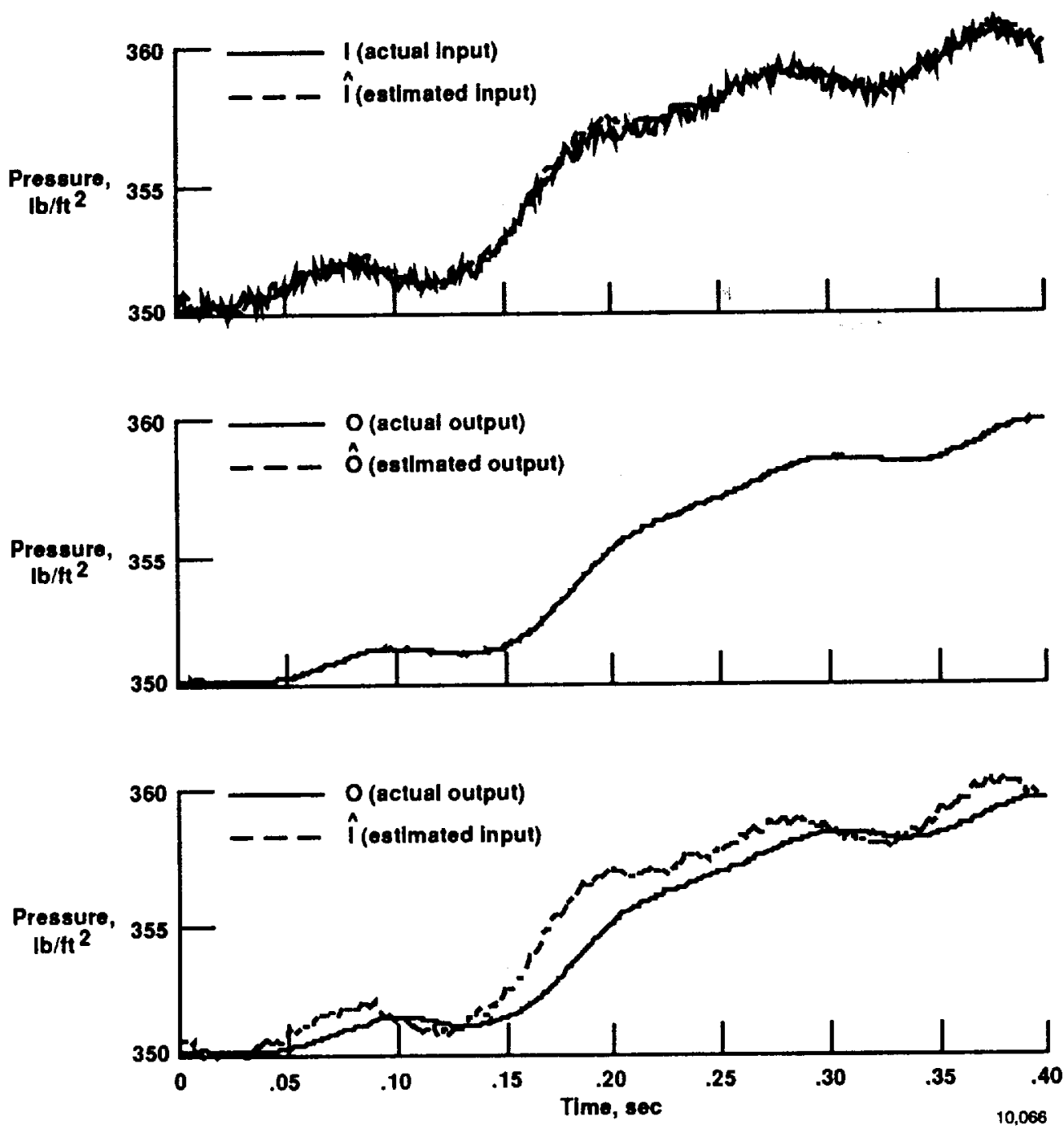

Fig. 12 Flight maneuver time history comparison for the real-time deconvolution algorithm output for an overdamped system. 

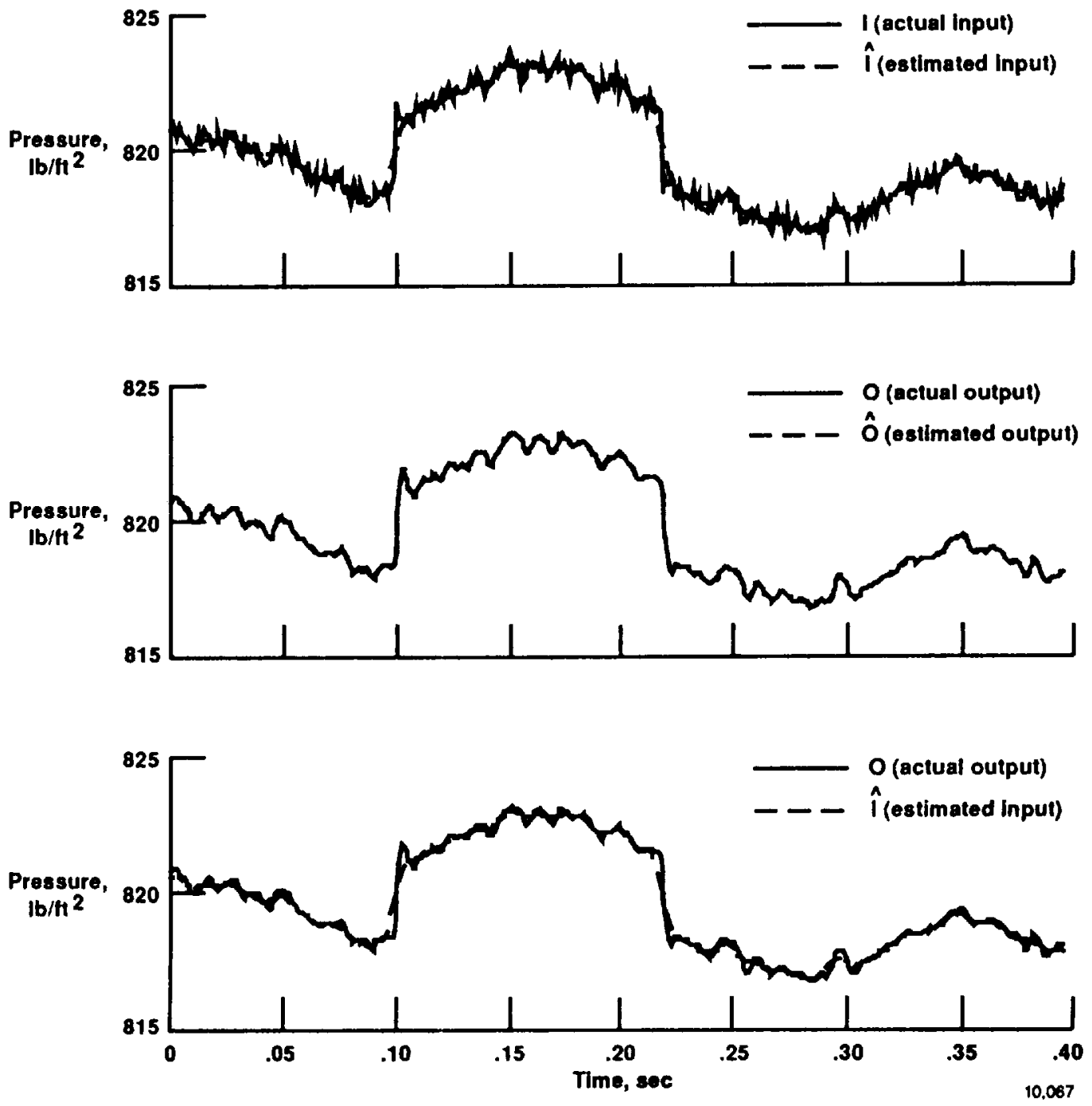

Fig. 13 Flight maneuver time history comparison for the postflight deconvolution algorithm for an underdamped system. 

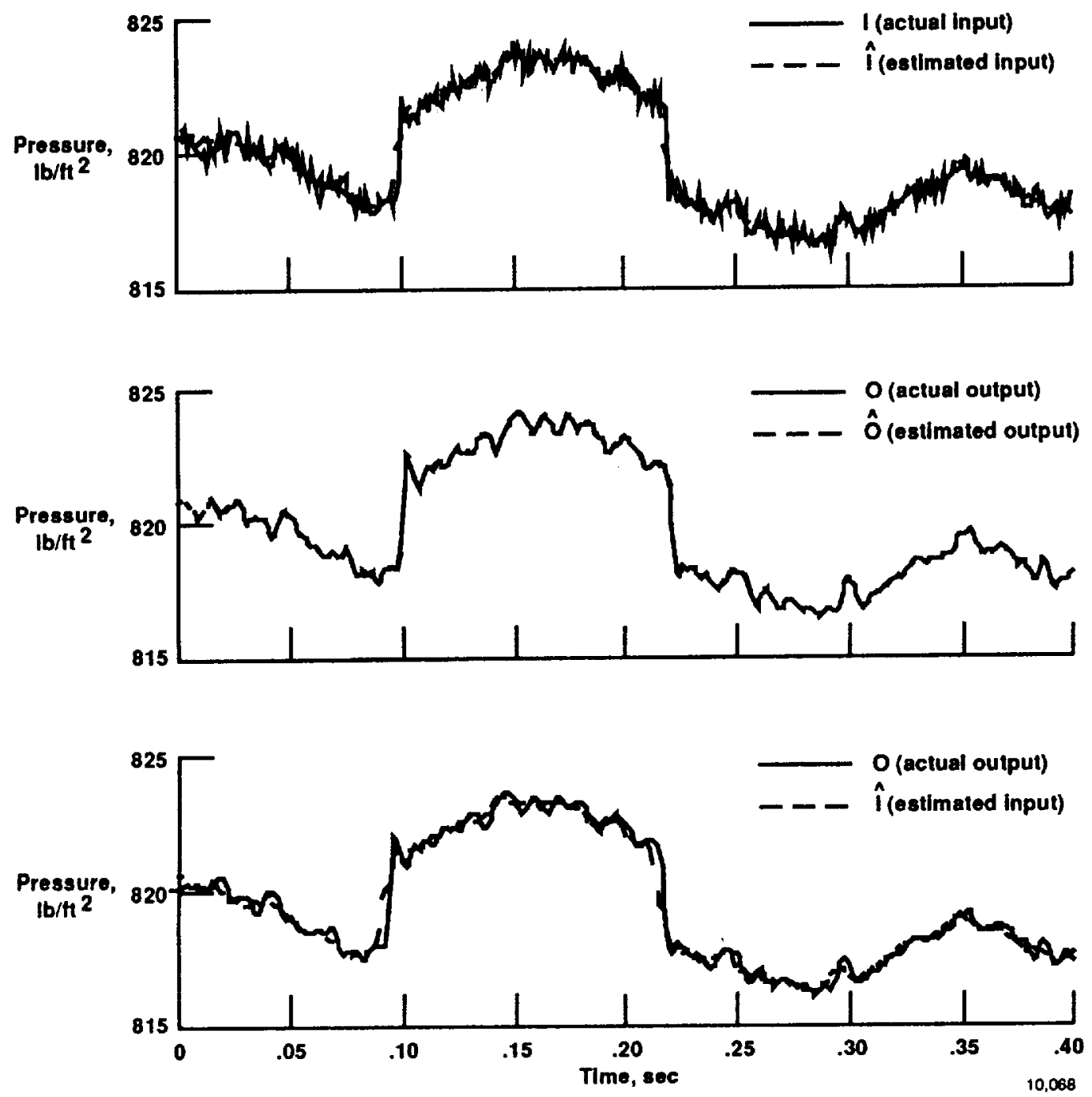

Fig. 14 Flight maneuver time history comparison for the real-time deconvolution algorithm for an underdamped system. 


\begin{tabular}{|c|c|c|c|c|}
\hline \multicolumn{5}{|c|}{ Report Documentation Page } \\
\hline $\begin{array}{l}\text { 1. Report No. } \\
\text { NASA TM-101716 }\end{array}$ & \multicolumn{2}{|c|}{ 2. Government Accossion No. } & \multicolumn{2}{|c|}{ 3. Recipient's Catalog No. } \\
\hline \multirow{2}{*}{\multicolumn{3}{|c|}{$\begin{array}{l}\text { 4. Titto and Subtitis } \\
\text { Compensating for Pneumatic Distortion in Pressure Sensing Devices }\end{array}$}} & \multicolumn{2}{|c|}{$\begin{array}{l}\text { 5. Report Date } \\
\text { January } 1990\end{array}$} \\
\hline & & & \multicolumn{2}{|c|}{ 6. Performing Organization Code } \\
\hline \multicolumn{3}{|l|}{ 7. Author(s) } & \multicolumn{2}{|c|}{$\begin{array}{l}\text { 8. Performing Organization Report No. } \\
\text { H-1586 }\end{array}$} \\
\hline \multicolumn{3}{|c|}{ Stephen A. Whitmore and Comelius T. Leondes } & \multicolumn{2}{|c|}{$\begin{array}{l}\text { 10. Work Unit No. } \\
\text { RTOP 505-68-71 }\end{array}$} \\
\hline \multicolumn{3}{|c|}{ 9. Porforming Orgenization Namo and Addreses } & \multirow{2}{*}{\multicolumn{2}{|c|}{ 11. Contract or Grant No. }} \\
\hline \multirow{2}{*}{\multicolumn{3}{|c|}{$\begin{array}{l}\text { NASA Ames Research Center } \\
\text { Dryden Flight Research Facility } \\
\text { P.O. Box 273, Edwards, CA } 93523-5000\end{array}$}} & & \\
\hline & & & \multicolumn{2}{|c|}{$\begin{array}{l}\text { 13. Type of Report and Period Covered } \\
\text { Technical Memorandum }\end{array}$} \\
\hline \multicolumn{3}{|c|}{$\begin{array}{l}\text { National Aeronautics and Space Administration } \\
\text { Washington, DC } 20546\end{array}$} & \multicolumn{2}{|c|}{ 14. Sponsoring Agency Code } \\
\hline \multicolumn{5}{|c|}{$\begin{array}{l}\text { Prepared as AIAA 90-0631 for presentation at AIAA 28th Aerospace Sciences Mecting, Reno, Nevada, } \\
\text { January 8-11, } 1990 .\end{array}$} \\
\hline \multicolumn{5}{|c|}{$\begin{array}{l}\text { 16. Abetract } \\
\text { In this paper a technique of compensating for pneumatic distortion in pressure sensing devices is devel- } \\
\text { oped and verified. This compensation allows conventional pressure sensing technology to obtain improved } \\
\text { unsteady pressure measurements. Pressure distortion caused by frictional attenuation and pneumatic reso- } \\
\text { nance within the sensing system makes obtaining unstcady pressure measurements by conventional sensors } \\
\text { difficult. Most distortion occurs within the pncumatic tubing which transmits pressure impulses from the air- } \\
\text { craft's surface to the measurement transducer. To avoid pneumatic distortion, experiment designers mount } \\
\text { the pressure sensor at the surface of the aircraft, (called in situ mounting). In situ transducers cannot always } \\
\text { fit in the available space and sometimes pncumatic tubing must be run from the aircraft's surface to the } \\
\text { pressure transducer. Research began at the National Aeronautics and Space Administration Ames Research } \\
\text { Center Dryden Flight Research Facility, supported by the University of California, Los Angeles, to develop } \\
\text { a technique to measure unstcady pressure data using conventional pressure sensing technology. A pneumatic } \\
\text { distortion model is reduced to a low-order, state-variable model retaining most of the dynamic characteris- } \\
\text { tics of the full model. The reduced-order model is coupled with results from minimum variance estimation } \\
\text { theory to develop an algorithm to compensatc for the effects of pneumatic distorion. Both postflight and } \\
\text { real-time algorithms are developed and evaluated using simulated and flight data. }\end{array}$} \\
\hline \multicolumn{2}{|c|}{$\begin{array}{l}\text { 17. Key Words (Suggeatod by Author(a)) } \\
\text { Lag compensation; Pneumatic distortion; } \\
\text { Pneumatic lag; Pressurc mcasurements; } \\
\text { Pressure sensors }\end{array}$} & $\begin{array}{r}\text { 18. Distribution } \mathrm{S} \\
\text { Unclass }\end{array}$ & — Unlimited & 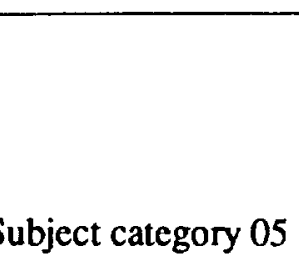 \\
\hline $\begin{array}{l}\text { 19. Socurity Classit. (of this roport) } \\
\text { Unclassified }\end{array}$ & $\begin{array}{r}\text { 20. Security } \\
\text { Uncl }\end{array}$ & his pegel & $\begin{array}{l}\text { 21. No. of pages } \\
21\end{array}$ & $\begin{array}{r}\text { 22. Price } \\
\mathrm{A} 02\end{array}$ \\
\hline
\end{tabular}




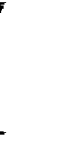

